\title{
The role of the Serratia marcescens SdeAB multidrug efflux pump and TolC homologue in fluoroquinolone resistance studied via gene-knockout mutagenesis
}

\author{
Sanela Begic and Elizabeth A. Worobec
}

Correspondence

Elizabeth A. Worobec

eworobe@cc.umanitoba.ca

Received 13 August 2007

Revised 22 October 2007

Accepted 12 November 2007

\author{
Department of Microbiology, University of Manitoba, Winnipeg, MB R3T 2N2, Canada
}

\begin{abstract}
Serratia marcescens is a prominent opportunistic nosocomial pathogen resistant to several classes of antibiotics. The major mechanism for fluoroquinolone resistance in various Gram-negative pathogens is active efflux. Our group previously identified SdeAB, a resistance-nodulation-cell division (RND) efflux pump complex, and a TolC-like outer-membrane protein (HasF), which together mediate energy-dependent fluoroquinolone efflux. In addition, a regulatory protein-encoding gene in the upstream region of $s d e A B$ was identified $(s d e R)$ and found to be $40 \%$ homologous to MarA, an Escherichia coli transcriptional regulator. To provide conclusive evidence as to the role of these components in $S$. marcescens, sdeB, has $F$ and sdeR deletion mutants were constructed. Suicide vectors were created and introduced via triparental mating into S. marcescens UOC-67 (wild-type) and, for sdeB and hasF, T-861 (clinical isolate). We have analysed these genetically altered strains using minimal inhibitory concentration (MIC) assays for a wide range of compounds (fluoroquinolones, SDS, novobiocin, ethidium bromide and chloramphenicol). Intracellular accumulation of a variety of fluoroquinolones was measured fluorospectroscopically. The $s d e B$, has $F$ and $s d e R$ knockout strains were consistently more susceptible to antibiotics than the parent strains, with the sdeB/has $F$ double knockout strain showing the highest susceptibility. A marked increase in fluoroquinolone (ciprofloxacin) accumulation was observed for strains deficient in either the $s d e B$ or has $F$ genes when compared to the parental strains, with the highest ciprofloxacin accumulation observed for the sdeB/has $F$ double knockout. Antibiotic accumulation assays for the sdeB knockout mutant strains performed in the presence of carbonyl cyanide $m$-chlorophenylhydrazone (CCCP), a proton-motive-force inhibitor, demonstrated that SdeAB-mediated efflux is proton-motive-force dependent. Due to the comparable susceptibility of the $s d e B$ and the has $F$ individual knockouts, we conclude that $S$. marcescens HasF is the sole outer-membrane component of the SdeAB pump. In addition, MIC data for sdeR-deficient and overexpressing strains confirm that $\mathrm{SdeR}$ is an activator of $s d e A B$ and acts to enhance the overall multidrug resistance of $S$. marcescens.
\end{abstract}

\section{INTRODUCTION}

Serratia marcescens is a prominent opportunistic pathogen, responsible for serious infections in immunocompromised individuals due primarily to its high intrinsic antibiotic resistance. It has been shown to be resistant to all major classes of antibiotics used, including fluoroquinolones, making the treatment of infections very difficult (Lambert \& O'Grady, 1992; Aucken \& Pitt, 1998; Fujimaki et al., 1989). The major mechanism for fluoroquinolone resistance in various Gram-negative organisms, including S. marcescens, is the active efflux of the antibiotic molecule mediated

Abbreviations: CCCP, carbonyl cyanide $m$-chlorophenylhydrazone; RND, resistance-nodulation-cell division. by efflux pumps belonging to the resistance-nodulation-cell division (RND) family. RND pumps work in conjunction with a periplasmic protein and an outer-membrane protein (e.g. Escherichia coli TolC) to form a tripartite system (Li \& Nikaido, 2004, and references therein).

We have established active efflux as a resistance mechanism in S. marcescens, and presented molecular characterization of two different efflux pumps (Kumar \& Worobec, 2005a). Of these, we have demonstrated that the SdeAB RND pump is a multidrug efflux pump with a wide range of substrates, having a high degree of homology to the AcrAB pump of E. coli. The sdeAB operon consists of the sdeA gene, encoding a periplasmic fusion protein, and the $s d e B$ gene, encoding the transporter of the RND pump on the 
inner membrane. We have also reported on an outermembrane component called HasF (TolC homologue) involved in energy-dependent efflux of antimicrobial agents (Kumar \& Worobec, 2005b). Computer-generated analysis of the $S$. marcescens HasF revealed a very similar structure to that of E. coli TolC, having the channel-tunnel structure characteristic of outer-membrane components of RND efflux pumps. No other tolC homologue was found upon searching the $S$. marcescens genome (Kumar \& Worobec, 2005b; http://www.sanger.ac.uk/Projects/S_marcescens/).

Most of the efflux pumps characterized to date have a regulatory protein-encoding gene upstream from the pumpencoding genes (Alekshun \& Levy, 1997; Barbosa \& Levy, 2000; Hachler et al., 1991). Upstream from the $s d e A B$ locus is sdeR, a 405 bp ORF which is transcribed in the opposite direction to $s$ deAB (Kumar, 2004). At the amino acid level, SdeR is $40 \%$ homologous to the MarA protein of E. coli, a transcriptional activator of the AcrAB-TolC drug efflux pump (Alekshun \& Levy, 1997; Barbosa \& Levy, 2000; Hachler et al., 1991), and sdeR is $50 \%$ homologous to marA at the DNA level. Amino acid prediction and threedimensional structural prediction also showed similarity to the MarA protein of $E$. coli, with a high degree of conservation of the DNA-binding helices (results not shown).

In this study, we addressed the importance of the SdeAB pump and the TolC-like protein in fluoroquinolone resistance by constructing has $F$ and $s d e B$ gene knockouts. In addition, through knockout mutagenesis, we have demonstrated the importance of SdeR in the regulation of the expression of the SdeAB efflux pump system.

\section{METHODS}

Bacterial strains and plasmids. All strains and plasmids used in this study are outlined in Table 1. All bacterial strains were grown in Luria-Bertani agar (LB), Trypticase Soy Agar (TSA) and Tryptone Soy Broth (TSB) (BD Diagnostics Systems). The ampicillin-resistant strains (containing $\mathrm{pKS}(+)$, $\mathrm{pKS}: \mathrm{SdeB}, \mathrm{pKS}: \mathrm{HasF}, \mathrm{pKS}: \Delta \mathrm{HasF}$, pKS:SdeR) were grown on LB plates and in LB broth containing $100 \mu \mathrm{g}$ ampicillin $\mathrm{ml}^{-1}$ (Sigma-Aldrich). The kanamycin-resistant strains (with pKIXX, pKS:SdeR: $\mathrm{Km}^{\mathrm{r}}$, pKS:SdeB: $\mathrm{Km}^{\mathrm{r}}$ ) were grown on LB plates and in LB broth containing $25 \mu \mathrm{g}$ kanamycin $\mathrm{ml}^{-1}$ (Sigma-Aldrich). The streptomycin-resistant strains (with pKNG101, pKNGsdeB, pKNGhasF, pKNGsdeR) were grown on LB plates and in LB broth containing $50 \mu \mathrm{g}$ streptomycin $\mathrm{ml}^{-1}$ (Sigma-Aldrich). The carbenicillin-resistance strains (with pEX1.8, pEXSH, pEXH, pEXS and pEXR) were grown on LB plates and in LB broth containing $300 \mu \mathrm{g}$ carbenicillin $\mathrm{ml}^{-1}$ (Sigma-Aldrich). Strains SDEAB1, SDEAB2, HASF100, HASF200, SDEAB3/HASF300 and SDER1 were grown on LB plates and in LB broth containing $100 \mu \mathrm{g}$ ampicillin $\mathrm{ml}^{-1}$ and $50 \mu \mathrm{g}$ streptomycin $\mathrm{ml}^{-1}$. UOC-67, MT616, CC118 and T861 were grown in the absence of any antibiotic.

Construction of S. marcescens hasF-, sdeB- and sdeR-deficient strains. Unique HincII/KpnI restriction sites within pKS:HasF (Kumar \& Worobec, 2005b) separated by approximately 700 bases were identified to be a feasible target for creating a deletion mutation in the has $F$ coding region. After transformation into S. marcescens UOC67 , the mutant clone was confirmed by restriction digestion, PCR and sequencing. The altered has $F$ fragment was then transferred into the
pKNG101 replacement vector and cloned into E. coli CC118 for stability. pKNG101 is a suicide vector that contains a conditional origin of replication (oriR6K), the $\operatorname{str} A B$ genes encoding streptomycin phosphotransferase $\left(\mathrm{Str}^{\mathrm{r}}\right)$, an origin of transfer (mobRK2), the $s a c B$ gene mediating sucrose sensitivity, and multiple cloning sites (Kaniga et al., 1991). The sdeB gene (Kumar \& Worobec, 2005a) was recloned as a $1.7 \mathrm{~kb} E c o \mathrm{RI} / B g l \mathrm{II}$ fragment into pKS, and transformed into UOC-67 in order to be disrupted by the insertion of a kanamycin-resistance cassette into a unique PstI restriction site. The sdeR gene (GenBank accession no. AY623133) was recloned as a $0.4 \mathrm{~kb} B a m \mathrm{HI}-E c o \mathrm{RI}$ fragment into pKS, and transformed into UOC-67 in order to be disrupted by the insertion of a kanamycin-resistance cassette into a unique EcoRV restriction site. The disrupted $s d e B$ and $s d e R$ fragments were transferred into the pKNG101 replacement vector and cloned into E. coli CC118 in a similar fashion as for hasF. Conjugation between the E. coli strain harbouring each of the disrupted $S$. marcescens $s d e B$, hasF and sdeR genes and the wild-type S. marcescens strain was carried out using E. coli helper strain MT616 (Finan et al., 1986) to create SDEAB1, HASF100 and SDER1, respectively. Conjugation was also carried out between the $E$. coli strain harbouring each of the disrupted $S$. marcescens $s d e B$ and has $F$ genes and the $S$. marcescens clinical isolate strain (T-861), using E. coli helper strain MT616 (Finan et al., 1986) for comparison purposes. For the double sdeB/hasF-deficient mutant (SDEAB3/HASF300), conjugation was carried out between the $S$. marcescens sdeB (SDEAB1)- and hasF (HASF100)-deficient strains, using E. coli MT616 as a helper. Analysis of transconjugants was based on sucrose sensitivity due to the synthesis of lethal levano compounds, catalysed by levanosucrase, the product of the $s a c B$ gene. All mutations were confirmed by restriction digestion, sequence analysis of both strands (National Research Council, Plant Biotechnology Institute, Saskatoon, Canada) and SDS-PAGE protein analysis.

Complementation of sdeB-, hasF- and sdeR-deficient strains. For complementation purposes, the expression vector pEX1.8 was used for cloning a $0.7 \mathrm{~kb}$ wild-type HincII/KpnI hasF fragment (pEXH), a $1.7 \mathrm{~kb}$ wild-type EcoRI/BglII $s d e B$ fragment (pEXS) and a $0.4 \mathrm{~kb}$ wildtype BamHI/EcoRI sdeR fragment (pEXR). In addition, $h a s F$ and $s d e B$ were introduced into SDEAB3/HASF300 (sdeB/hasF-deficient strain). To complement both has $F$ and $s d e B$ simultaneously, the $1.7 \mathrm{~kb} E c o \mathrm{RI} /$ $B g l I I s d e B$ fragment was cloned into the EcoRI/BglII site, and the $0.7 \mathrm{~kb}$ hasF HincII/KpnI fragment was cloned into the HincII/KpnI site of pEX1.8 (pEXSH). To construct strain SM2000, pEXH was first transferred into the UOC-67 by electroporation. Conjugation between UOC-67/pEXH harbouring the wild-type hasF gene and SDEAB3/ HASF300 (sdeB/hasF-deficient) was carried out using the E. coli helper strain. The same two-step procedure was used to transform pEXS and pEXSH into SDEAB3/HASF300 and pEXR into SDER1 to create SM3000, SM1000 and SDER2, respectively. Selection of transconjugants was achieved by incorporation of ampicillin and streptomycin into the growth medium. Transformants were verified by restriction digestion and sequence analysis of both strands of the wild-type genes (National Research Council, Plant Biotechnology Institute, Saskatoon, Canada). Proteins were expressed under the control of the $\mathrm{P}_{\text {tac }}$ promoter on pEX1.8 and induced by the addition of varying concentrations (0.5$5 \mathrm{mmol}^{-1}$ ) of IPTG (Sigma-Aldrich) to the growth medium.

sdeR overexpression. A $0.4 \mathrm{~kb}$ BamHI-EcoRI sdeR PCR fragment (including the entire open reading frame and ribosome-binding site) was cloned into the BamHI/EcoRI site of pEX1.8 (pEXR) and transformed into UOC-67 to generate SDER3. pEX1.8 alone was also transformed into UOC-67 to generate SDER4 to use as a control. Transformants were confirmed via restriction digestion and sequence analysis.

Antibiotic susceptibility tests. Susceptibility of SDEAB1, SDEAB2, HASF100, HASF200, SDEAB3/HASF300, SM1000, SM2000, SM3000, SDER1, SDER2, SDER3 and SDER4 to norfloxacin, ciprofloxacin, 
Table 1. Bacterial strains and plasmids used in the study

Abbreviations: Kan, kanamycin; Amp, ampicillin; Str, streptomycin; Car, carbenicillin.

\begin{tabular}{|c|c|c|}
\hline Strain or plasmid & Characteristic & Source/reference \\
\hline \multicolumn{3}{|l|}{ E. coli } \\
\hline MT616 & MT607 (pro-82 thi-1 hsdR17 supE44):pRK600 & Finan et al. (1986) \\
\hline CC118 & $\begin{array}{l}\operatorname{araD139} \Delta(\text { ara leu }) 7697 \Delta \text { lacX74 phoA20 galE galK thi rpsE rpoB } \\
\text { argEam recA1 }\end{array}$ & $\begin{array}{l}\text { C. Manoil, University of Washington, } \\
\text { Seattle, USA }\end{array}$ \\
\hline \multicolumn{3}{|l|}{ S. marcescens } \\
\hline UOC-67 & Wild-type, ATCC 13880 & American Type Culture Collection \\
\hline $\mathrm{T}-861$ & Clinical isolate & $\begin{array}{l}\text { D. Hoban, Health Science Centre, } \\
\text { Winnipeg }\end{array}$ \\
\hline SDEAB1 & sdeB-deficient UOC-67 & This study \\
\hline SDEAB2 & sdeB-deficient T-861 & This study \\
\hline HASF100 & hasF-deficient UOC-67 & This study \\
\hline HASF200 & hasF-deficient T-861 & This study \\
\hline SDEAB3/HASF300 & sdeB/hasF-deficient UOC-67 & This study \\
\hline SDER1 & sdeR-deficient UOC-67 & This study \\
\hline SDER2 & SDER1/pEXR & This study \\
\hline SM3000 & SDEAB3/HASF300 with pEXS & This study \\
\hline SM2000 & SDEAB3/HASF300 with pEXH & This study \\
\hline SM1000 & SDEAB3/HASF300 with pEXSH & This study \\
\hline SDER3 & UOC-67/pEXR & This study \\
\hline SDER4 & UOC-67/pEX1.8 & This study \\
\hline \multicolumn{3}{|l|}{ Plasmids } \\
\hline pKS $(+)$ & ColE1 replicon, $A m p^{r}$, lac $Z^{\prime}$ & Stratagene \\
\hline pKS : SdeB & pKS with $1.7 \mathrm{~kb} E c o \mathrm{RI} / B g l \mathrm{I} s d e B$ fragment & This study \\
\hline pKS:SdeB:Km ${ }^{r}$ & pKS: SdeB with $1.4 \mathrm{~kb} \mathrm{Kan}^{\mathrm{r}}$ insertion in $s d e B$ & This study \\
\hline pKS : HasF & pKS with $1.5 \mathrm{~kb} E c o \mathrm{RI} / B a m \mathrm{HI}$ hasF fragment & This study \\
\hline $\mathrm{pKS}: \Delta \mathrm{HasF}$ & pKS: HasF with HincII/KpnI 700 bp deleted & This study \\
\hline pKS : SdeR & pKS with 400-base BamHI/EcoRI sdeR fragment & This study \\
\hline pKS:SdeR: Km ${ }^{r}$ & pKS : SdeR with a $1.4 \mathrm{~kb} \mathrm{Kan}^{\mathrm{r}}$ insertion in $s d e R$ & This study \\
\hline pEX1.8 & CoE1 replicon, $\mathrm{Amp}^{\mathrm{r}}$ Car $^{\mathrm{r}}$, carries $l a c I^{\mathrm{Q}}$ and a $\mathrm{P}_{\text {tac }}$ promoter & Pearson et al. (1997) \\
\hline pEXSH & pEX1.8 with wild-type $700 \mathrm{bp}$ has $F$ and $1.7 \mathrm{~kb} s d e B$ fragments & This study \\
\hline pEXH & pEX1.8 with wild-type 700 bp hasF fragment & This study \\
\hline pEXS & pEX1.8 with wild-type $1.7 \mathrm{~kb} s d e B$ fragment & \\
\hline pEXR & pEX1.8 with $405 \mathrm{bp}$ BamHI/EcoRI sdeR fragment & This study \\
\hline pKNG101 & Suicide vector, pir oriR6K mobRK2 sacB $\mathrm{Str}^{\mathrm{r}}$ & Kaniga et al. (1991) \\
\hline pKNGsdeB & pKNG101 with $3.1 \mathrm{~kb}$ insertionally disrupted $s d e B$ fragment & This study \\
\hline pKNGhasF & pKNG101 with 800 bp deleted hasF & This study \\
\hline pKNGsdeR & pKNG101 with $1.8 \mathrm{~kb}$ insertionally disrupted $s d e R$ fragment & This study \\
\hline pKIXX & $1.4 \mathrm{~kb} \mathrm{Kan}{ }^{\mathrm{r}}$ cassette flanked by $S m a \mathrm{I}$ & Pharmacia PL Biochemicals \\
\hline
\end{tabular}

ofloxacin, chloramphenicol, novobiocin, SDS and ethidium bromide (Sigma-Aldrich) was tested using the minimum inhibitory concentration (MIC) twofold broth dilution method (CLSI, 2006). Overnight cultures in TSB were diluted 1000-fold in fresh broth, grown at $37^{\circ} \mathrm{C}$ to $\mathrm{OD}_{600} 0.5-0.9$ and $5 \mu \mathrm{l}$ of the bacterial suspension was inoculated in TSB containing serial dilutions of each antibiotic. Results are reported as MIC, the concentration of antibiotic that inhibited visible growth determined by absence of turbidity in TSB after $18 \mathrm{~h}$ shaking incubation at $37^{\circ} \mathrm{C}$.

Fluoroquinolone accumulation. The accumulation of ciprofloxacin by SDEAB1, SDEAB2, HASF100, HASF200, SDEAB3/HASF300, SM1000, SM2000 and SM3000 was measured using the method of Mortimer \& Piddock (1991). Cultures were grown until the $\mathrm{OD}_{600}$ reached 0.5-0.7. Cells were harvested by centrifugation at $4000 \mathrm{~g}$ for $15 \mathrm{~min}$ at room temperature, suspended in PBS pH 7.5 and washed twice. Pellets were suspended in 1/10 volume of PBS. Ciprofloxacin was added to $1 \mathrm{ml}$ aliquots to reach $10 \mu \mathrm{g} \mathrm{ml}^{-1}$ external concentration and allowed to incubate at room temperature for 1-12 min. Carbonyl cyanide $m$-chlorophenylhydrazone (CCCP) (SigmaAldrich) was added to a final concentration of $100 \mu \mathrm{M}$ after $5 \mathrm{~min}$ antibiotic incubation. After incubation, a rapid centrifugation (13000 $\mathrm{g}$ at $4{ }^{\circ} \mathrm{C}$ for $1 \mathrm{~min}$ ) was carried out, followed by a wash in cold PBS, suspension in $1 \mathrm{ml} 0.1 \mathrm{M}$ glycine. $\mathrm{HCl} \mathrm{pH} 3.0$ to lyse the cells, and overnight incubation at room temperature. This suspension was centrifuged at $11000 \mathrm{~g}$ for $5 \mathrm{~min}$ to remove cellular debris and antibiotic concentration measured in a Shimadzu RF-1501 spectrofluorometer. The fluorescence of ciprofloxacin was measured at $279 \mathrm{~nm}$ excitation wavelength and $447 \mathrm{~nm}$ emission wavelength. Ciprofloxacin concentration was calculated using a standard curve (concentration ranging from 100 to $1000 \mathrm{ng}$ ) in $0.1 \mathrm{M}$ glycine. $\mathrm{HCl}$ $\mathrm{pH}$ 3.0. The results were expressed as nanograms of ciprofloxacin incorporated per milligram (dry weight) of bacteria. Results in the figures are means and standard errors. 


\section{RESULTS}

\section{Antibiotic susceptibility tests}

The most dramatic increase in antibiotic susceptibility was found with the double-knockout strain (SDEAB3/ HASF300) in comparison to UOC-67 (Table 2). For SDEAB1 and HASF100, susceptibility to all compounds tested increased in comparison to UOC-67, and for SDEAB2 and HASF200 in comparison to T-861. MIC values for HASF100 were identical to those of SDEAB1 for all compounds tested, except for chloramphenicol, where there was a slight difference. A similar trend was found for HASF200 and SDEAB2. MIC values for SM1000 (hasF/sdeB-complemented strain), SM2000 (hasFcomplemented strain) and SM3000 (sde-complemented strain) were identical to those of wild-type UOC-67 for all antibiotics tested, suggesting that both $\mathrm{SdeB}$ and HasF are equally important for $S$. marcescens multidrug resistance.

The sdeR-overexpressing strain (SDER3) was considerably more resistant than either UOC-67 or SDER4 (UOC-67/ pEX1.8) (Table 2) for all compounds tested, while SDER1 (sdeR-deficient) was more susceptible to all compounds. Complementation of SDER1 with sdeR (SDER2) resulted in a restoration of resistance to wild-type levels.

\section{Fluoroquinolone accumulation}

The loss of the functioning efflux pump SdeAB (SDEAB1 and SDEAB2) resulted in a significant increase in ciprofloxacin accumulation as compared to UOC-67 (Fig. 1a) and T-861 parental strains, respectively (Fig. 1b).
The addition of CCCP, a proton-motive-force inhibitor, increased the accumulation rate of UOC-67 (Fig. 1a) and T-861 (Fig. 1b); however, it had no effect upon ciprofloxacin accumulation in the knockout mutant strains. Ciprofloxacin accumulation for the SM3000 (sdeB-complemented) strain was very similar to that of the UOC-67 in the absence and presence of CCCP.

The loss of the functioning hasF gene (HASF100 and HASF200) also resulted in a notable increase in ciprofloxacin accumulation as compared to UOC-67 (Fig. 2a) and T-861 parental strains, respectively (Fig. 2b). As expected, the addition of CCCP increased the accumulation of UOC-67 (Fig. 2a) and T-861 (Fig. 2b); however, it had no effect upon ciprofloxacin accumulation in the HASF100 and HASF200 knockout mutant strains. Ciprofloxacin accumulation for the SM2000 (hasF complemented) strain was very similar to that of the UOC-67 in the absence and presence of CСCP.

Although the accumulation of SDEAB1 and HASF100 was almost identical, the loss of both hasF and sdeB (SDEAB3/ HASF300) resulted in increased ciprofloxacin accumulation (a $30 \%$ increase in SDEAB3/HASF100 as compared to SDEAB1 or HASF100 alone) (Fig. 3). Addition of CCCP resulted in a further increase in ciprofloxacin accumulation for the double knockout strain (SDEAB3/HASF300) resulting in a $40 \%$ increase in accumulation as compared to HASF100 in the absence of CCCP (Fig. 3). SM1000 (sdeB/hasF-complemented) in the absence of CCCP showed the lowest accumulation, lower than that of either SDEAB1 or HASF100 alone, suggesting that $s d e B$ and hasF have a synergistic effect. In the presence of CCCP, SM1000 showed a significant increase in accumulation.

Table 2. Susceptibilities of wild-type, clinical isolate and various $s d e B$ and has $F$ mutant $S$. marcescens strains to a range of antibiotics

Abbreviations: Cip, ciprofloxacin; Nor, norfloxacin; Ofl, ofloxacin; Chl, chloramphenicol; Nov, novobiocin; SDS, sodium dodecyl sulphate; EtBr, ethidium bromide. Results are representative of three trials.

\begin{tabular}{|c|c|c|c|c|c|c|c|}
\hline Strain & \multicolumn{7}{|c|}{$\operatorname{MIC}\left(\mu \mathrm{g} \mathrm{ml}^{-1}\right)$} \\
\hline T-861 (clinical isolate) & 128 & 128 & 128 & 256 & 256 & 4 & 8 \\
\hline SDEAB1 (sdeB-deficient in UOC-67) & 0.125 & 0.125 & 0.125 & 4 & 16 & 0.25 & 0.25 \\
\hline SDEAB2 (sdeB-deficient in T-861) & 0.008 & 0.008 & 0.008 & 16 & 32 & 0.25 & 0.25 \\
\hline SDEAB3/HASF300 (sdeB/hasF-deficient in UOC-67) & 0.002 & 0.002 & 0.002 & 0.004 & 0.016 & 0.004 & 0.004 \\
\hline SM3000 (sdeB-complemented SDEAB1) & 1 & 1 & 1 & 32 & 64 & 1 & 1 \\
\hline SM2000 (hasF-complemented HASF100) & 1 & 1 & 1 & 32 & 64 & 1 & 1 \\
\hline SM1000 (sdeB, hasF-complemented SDEAB3/HASF300) & 1 & 1 & 1 & 32 & 64 & 1 & 1 \\
\hline SDER1 (sdeR-deficient UOC-67) & 0.25 & 0.25 & 0.25 & 8 & 8 & 0.25 & 0.25 \\
\hline
\end{tabular}



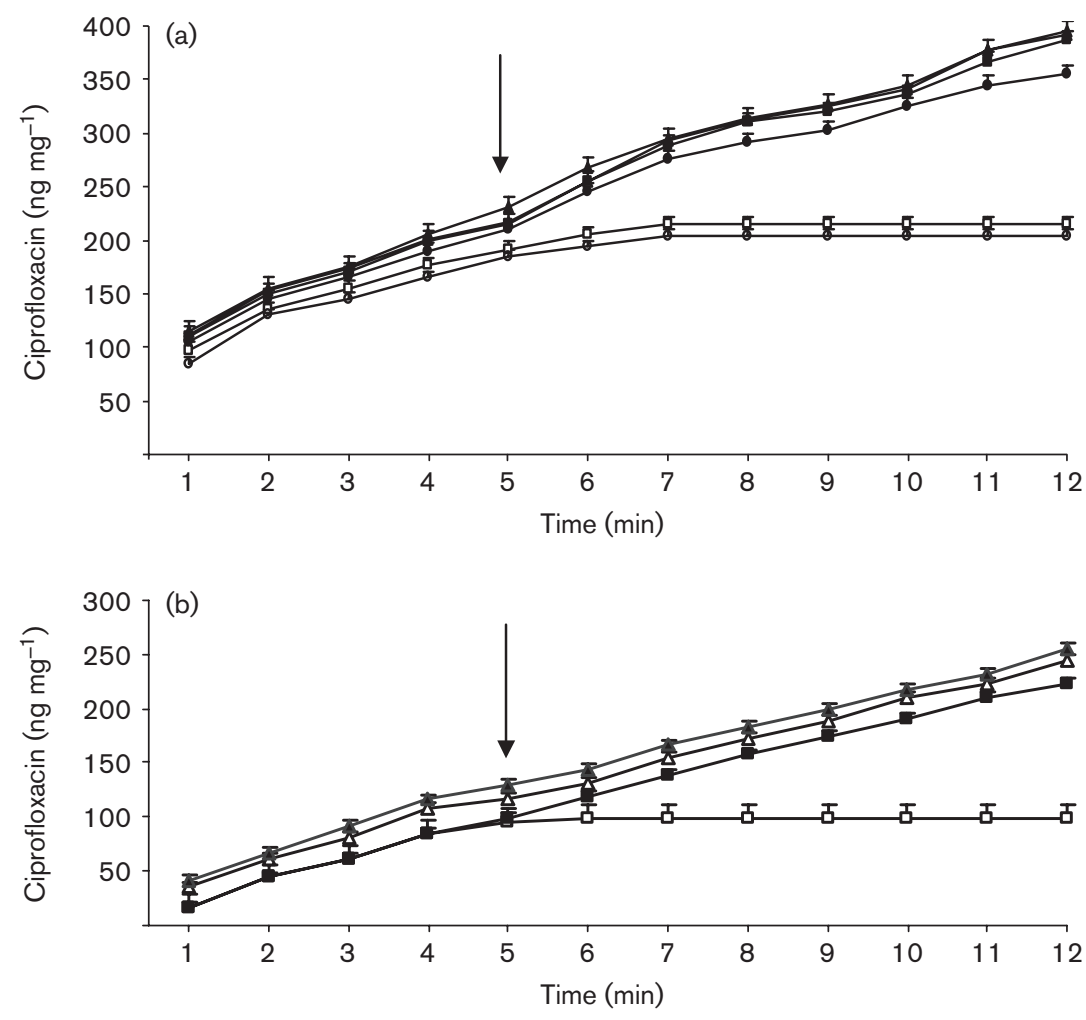

Fig. 1. Accumulation of ciprofloxacin by (a) wild-type, mutant and complemented sdeB $S$. marcescens UOC-67 and (b) clinical isolate and mutant sdeB S. marcescens T-861 in the absence and presence of CCCP. Various strains are depicted as follows: (a) UOC-67 in presence ( $\square$ ) and absence $(\square)$ of CCCP; SDEAB1 in presence $(\boldsymbol{A})$ and absence $(\triangle)$ of CCCP; SM3000 in presence (O) and absence $(\bigcirc)$ of CCCP; (b) T-861 in presence $(\boldsymbol{\square})$ and absence $(\square)$ of CCCP; SDEAB2 in presence $(\boldsymbol{\Lambda})$ and absence $(\triangle)$ of CCCP. The proton-motive-force uncoupler CCCP $(100 \mu \mathrm{M})$ was added after 5 min incubation (arrow). All trials were performed in triplicate.
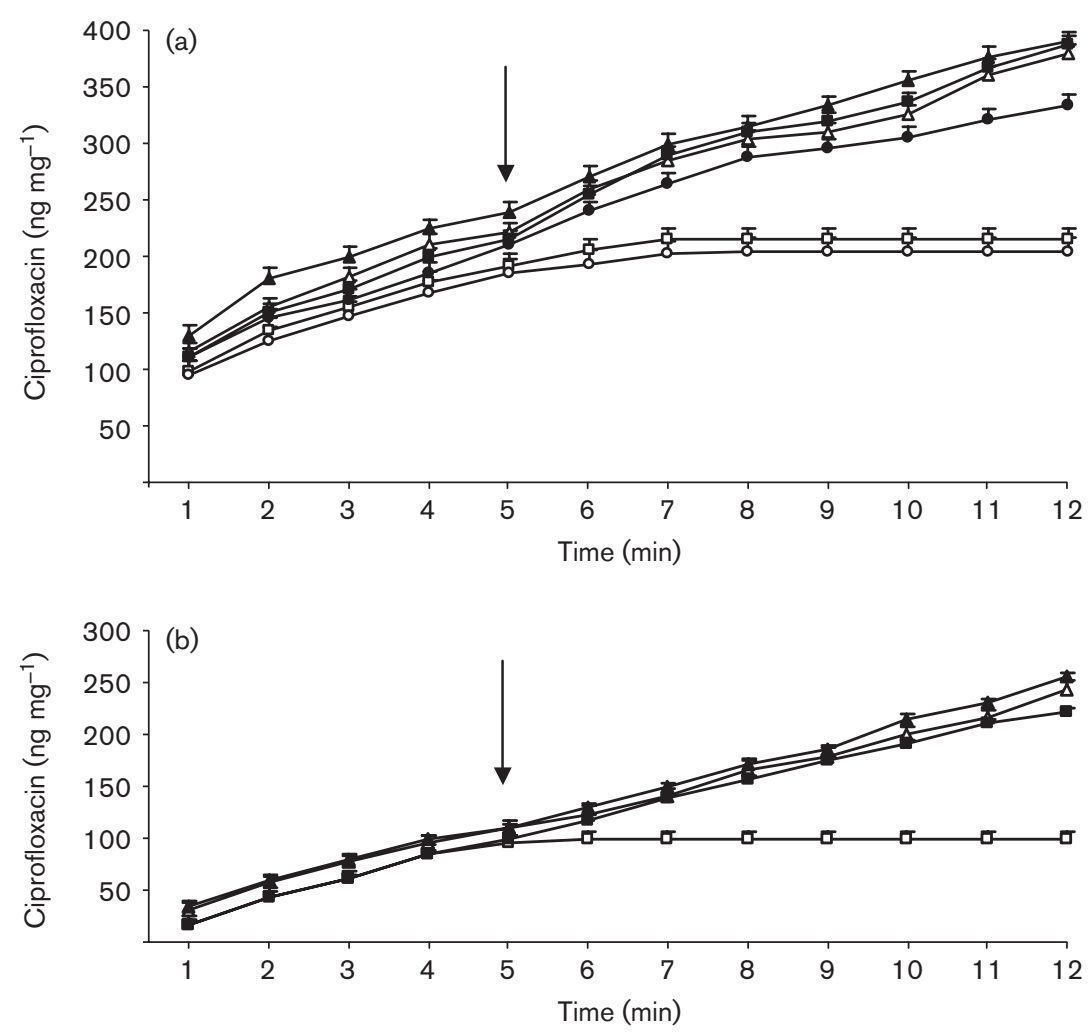

Fig. 2. Accumulation of ciprofloxacin by (a) wild-type, mutant and complemented has $F S$. marcescens UOC-67 and (b) clinical isolate and mutant hasF S. marcescens T-861 in the absence and presence of CCCP. Various strains are depicted as follows: (a) UOC-67 in presence $(\boldsymbol{\square})$ and absence $(\square)$ of CCCP; HASF100 in presence $(\boldsymbol{\Delta})$ and absence $(\triangle)$ of CCCP; SM2000 in presence (O) and absence $(\bigcirc)$ of CCCP; (b) T-861 in presence (ם) and absence ( $\square$ ) of CCCP; HASF200 in presence $(\boldsymbol{\Lambda})$ and absence $(\triangle)$ of CCCP. The proton-motive-force uncoupler $\mathrm{CCCP}$ $(100 \mu \mathrm{M})$ was added after 5 min incubation (arrow). All trials were performed in triplicate. 


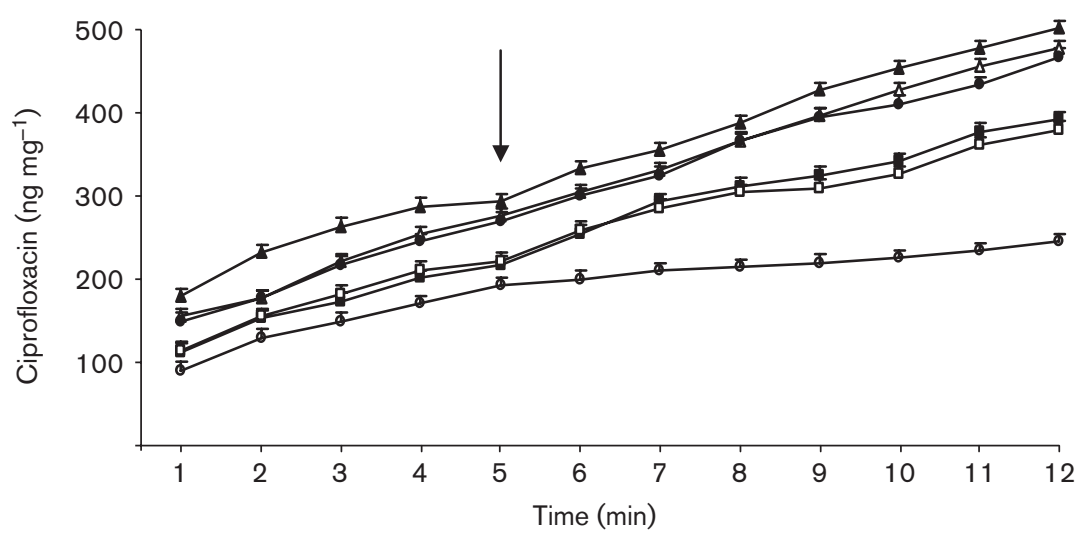

Fig. 3. Accumulation of ciprofloxacin by sdeB-deficient, hasF-deficient, double-deficient and complemented $S$. marcescens strains. SDEAB3/HASF300 accumulation in absence and presence of CCCP is shown, whereas the CCCP results for SDEAB1 and HASF100 can be found in Figs 1 and 2 . Various strains are depicted as follows: SDEAB1, no CCCP (ם); HASF100, no CCCP ( $\square$ ); SDEAB3/HASF300, in absence $(\triangle)$ and presence $(\mathbf{A})$ of CCCP; SM1000, in absence $(\bigcirc)$ and presence $(\boldsymbol{O})$ of CCCP. The proton-motive-force uncoupler CCCP $(100 \mu \mathrm{M})$ was added after $5 \mathrm{~min}$ incubation (arrow). All trials were performed in triplicate.

\section{DISCUSSION}

Three different RND pumps have been identified in $S$. marcescens: SdeAB, SdeCDE (Kumar \& Worobec, 2005a) and SdeXY (Chen et al., 2003). SdeCDE appears to be a homologue of the E. coli MdtABC system (Kumar \& Worobec, 2005a). Preliminary results thus far demonstrate that SdeCDE is a very selective pump and provides very limited drug resistance, with novobiocin being its only substrate (data not shown). Novobiocin is an inhibitor of DNA gyrase that targets the GyrB subunit (Maxwell \& Lawson, 2003). Similar results have been reported for MdtABC of E. coli (Nagakubo et al., 2002). SdeXY confers norfloxacin and tetracycline resistance and appears to be a close homologue of the E. coli AcrAB-TolC pump (Chen et al., 2003). Our study demonstrates that $S$. marcescens $S d e A B$ is the major pump, functioning with broad substrate specificity.

$s d e B$ and has $F$ knockouts resulted in an increase in susceptibility for all compounds tested, demonstrating that both has $F$ and $s d e B$ mutants were hypersusceptible to a range of antibiotics, dyes and detergents. This finding is consistent with Buckley et al. (2006), where the authors reported a similar antibiotic-susceptibility trend for $a c r B$ and tolC knockouts in Salmonella enterica, and with Ruzin et al. (2007), where the same finding was reported for an ade $B$ knockout strain (inner-membrane transporter of the AdeABC multidrug efflux pump homologous to AcrAB of E. coli). Hypersusceptibility to a variety of compounds was also reported for $a c r A B$ deletion mutants in E. coli (Ma et al., 1995); AcrAB is a pump to which S. marcescens $\mathrm{SdeAB}$ is closely related. Similarly, in Helicobacter pylori, knockout mutant strains in which TolC homologues were inactivated displayed higher susceptibility to antibiotics and ethidium bromide (van Amsterdam et al., 2005), similar to the hasF knockout strains. Also, in Brucella suis, mutations in bepC, encoding a TolC homologue, increased the antimicrobial susceptibility, contributing to the intrinsic resistance/susceptibility phenotype (Posadas et al., 2007). Corresponding mutant strains complemented with $s d e B$ or hasF, or both, resulted in a restoration of resistance to wild-type levels, demonstrating the overall importance of both these components.

$s d e R$ knockout resulted in a decrease in resistance that was restored to wild-type levels upon complementation, while overexpression of $s d e R$ significantly increased resistance. These results demonstrate the role of SdeR as an activator of SdeAB expression. Similar results were found in Enterobacter aerogenes, where the RamA activator was shown to induce the expression of the efflux pump and act as an activator (Chollet et al., 2004). SdeR is $40 \%$ homologous to the MarA protein of E. coli, a transcriptional activator of the AcrAB-TolC drug efflux pump, and the amino acid sequences of the two DNA-binding motifs essential to the regulatory function of MarA are well conserved in SdeR. In addition to suggesting that SdeR and MarA may recognize the same set of operator sequences, we have detected the presence of a putative mar box in the $s d e R$ promoter that is well conserved according to the consensus (Kumar, 2004).

Strains in the study were examined spectrofluorimetrically for efflux of ciprofloxacin and other fluoroquinolones not reported (norfloxacin, ofloxacin, levofloxacin), before and after the addition of the uncoupler CCCP. Knockout mutant strains (SDEAB1, SDEAB2, HASF100, HASF200, SDEAB3/HASF300) that do not efflux antibiotics were not affected by the addition of CCCP. When the HasF outermembrane protein (HASF100, HASF200) or both HasF and the pump (SDEAB3/HASF300) were no longer produced, mutant strains continued to accumulate antibiotics in a steady fashion before and after the addition of the uncoupler (Figs 1, 2 and 3). In contrast, antibiotic accumulation of the wild-type strain UOC-67 and the clinical T-861 isolate was low, but increased after the addition of CCCP as the proton gradient-dependent efflux collapsed (Figs 1, 2 and 3). This is a similar finding to that reported by Pumbwe \& Piddock (2002), where the cmeB gene encoding a Campylobacter jejuni multidrug efflux pump was knocked out and mutant strains displayed higher ciprofloxacin accumulation than the wild-type $C$. jejuni. For strains SM3000 and SM2000, complemented 
with $s d e B$ and has $F$ genes, respectively, the accumulation trend was similar to that of UOC-67 where the levels increased after CCCP collapsed the gradient. This suggests that both components, SdeB and HasF, are important in the drug efflux as the accumulation levels for each are very similar to that of UOC-67. For the sdeB/hasF doublecomplemented strain SM1000, in the absence of CCCP, accumulation levels were lower than those of the corresponding uncomplemented mutants SDEAB1 or HASF100, suggesting that most of the compound is effluxed out of the cell through the restored outermembrane channel. With the addition of CCCP, SM1000 accumulation levels increased to that of SDEAB3/HASF300 in the absence of CCCP, suggesting that the collapse of the gradient did restore the accumulation once again (Fig. 3). SM100 accumulation levels, however, were similar in trend to UOC-67 (results not shown).

Our early studies show that the SdeCDE pump does not efflux ciprofloxacin (data not shown); however, the SdeXY pump has been reported to efflux fluoroquinolones (Chen et al., 2003), which could account for the minor change in the ciprofloxacin accumulation for the knockout mutant strains in the absence and presence of CCCP. Ciprofloxacin accumulation for the $s d e B$ and has $F$ knockout mutant strains (SDEAB1, HASF100) was very similar (Fig. 3) and both had a comparable susceptibility profile (Table 2), suggesting that the $S$. marcescens HasF is the only outermembrane component of efflux pumps in this organism.

In conclusion, using knockout mutagenesis, we have established the importance of the $S$. marcescens SdeAB multidrug efflux pump as the primary RND pump responsible for increasing $S$. marcescens resistance to a range of compounds. In addition, we have established the role of HasF in contributing to the intrinsic resistance of $S$. marcescens to a variety of substances and the role of SdeR as an activator of the SdeAB efflux pump.

\section{ACKNOWLEDGEMENTS}

We are grateful to T. de Kievit for her valuable suggestions, A. Kumar and J. Manuel for their generous assistance and A. Ducas and L. Larsen for their technical support. This work was supported by a Natural Sciences and Engineering Research Council of Canada operating grant (to E.A.W.). S. B. is the recipient of a University of Manitoba Graduate Student Fellowship.

\section{REFERENCES}

Alekshun, M. N. \& Levy, S. B. (1997). Regulation of chromosomally mediated multiple antibiotic resistance: the mar regulon. Antimicrob Agents Chemother 41, 2067-2075.

Aucken, H. M. \& Pitt, T. L. (1998). Antibiotic resistance and putative virulence factors of Serratia marcescens with respect to $\mathrm{O}$ and K serotypes. J Med Microbiol 47, 1105-1113.

Barbosa, T. M. \& Levy, S. B. (2000). Differential expression of over 60 chromosomal genes in Escherichia coli by constitutive expression of MarA. J Bacteriol 182, 3467-3474.
Buckley, A. M., Webber, M. A., Cooles, S., Randall, L. P., La Ragione, R. M., Woodward, M. J. \& Piddock, L. J. V. (2006). The AcrAB-TolC efflux system of Salmonella enterica serovar Typhimurium plays a role in pathogenesis. Cell Microbiol 8, 847-856.

Chen, J., Kuroda, T., Huda, M. N., Mizushima, T. \& Tsuchiya, T. (2003). An RND-type multidrug efflux pump SdeXY from Serratia marcescens. J Antimicrob Chemother 52, 176-179.

Chollet, R., Chevalier, J., Bollet, C., Pages, J. M. \& Davin-Regli, A. (2004). RamA is an alternate activator of the multidrug resistance cascade in Enterobacter aerogenes. Antimicrob Agents Chemother 48, 2518-2523.

CLSI (2006). Performance Standards for Antimicrobial Susceptibility Testing. Supplement M100-S16. Wayne, PA: Clinical and Laboratory Standards Institute.

Finan, T. M., Kunkel, B., De Vos, G. F. \& Signer, E. R. (1986). Second symbiotic megaplasmid in Rhizobium meliloti carrying exopolysaccharide and thiamine synthesis genes. J Bacteriol 167, 66-72.

Fujimaki, K., Fujii, T., Aoyama, H., Sato, K.-I., Inoue, Y., Inoue, M. \& Mitsuhashi, S. (1989). Quinolone resistance in clinical isolates of Serratia marcescens. Antimicrob Agents Chemother 33, 785-787.

Hachler, H., Cohen, S. P. \& Levy, S. B. (1991). marA, a regulated locus which controls expression of chromosomal multiple antibiotic resistance in Escherichia coli. J Bacteriol 173, 5532-5538.

Kaniga, K., Delor, I. \& Cornelis, G. R. (1991). A wide-host-range suicide vector for improving reverse genetics in Gram-negative bacteria: inactivation of the blaA gene of Yersinia enterocolitica. Gene 109, 137-141.

Kumar, A. (2004). Characterization of RND efflux pumps of Serratia marcescens. PhD thesis, Department of Microbiology, University of Manitoba.

Kumar, A. \& Worobec, E. A. (2005a). Cloning, sequencing and characterization of the SdeAB multidrug efflux pump of Serratia marcescens. Antimicrob Agents Chemother 49, 1495-1501.

Kumar, A. \& Worobec, E. A. (2005b). HasF, a TolC-homolog of Serratia marcescens, is involved in energy-dependent efflux. Can J Microbiol 51, 497-500.

Lambert, H. P. \& O'Grady, F. W. (1992). Antibiotics and Chemotherapy. Edinburgh: Churchill Livingstone.

Li, X.-Z. \& Nikaido, H. (2004). Efflux-mediated drug resistance in bacteria. Drugs 64, 159-204.

Ma, D., Cook, D. N., Alberti, M., Pon, N. G., Nikaido, H. \& Hearst, J. E. (1995). Genes acrA and $a c r B$ encode a stress-induced efflux system of Escherichia coli. Mol Microbiol 16, 45-55.

Maxwell, A. \& Lawson, D. M. (2003). The ATP-binding site of type II topoisomerases as a target for antibacterial drugs. Curr Top Med Chem 3, 283-303.

Mortimer, P. G. \& Piddock, L. J. (1991). A comparison of methods used for measuring the accumulation of quinolones by Enterobacteriaceae, Pseudomonas aeruginosa and Staphylococcus aureus. J Antimicrob Chemother 28, 639-653.

Nagakubo, S., Nishino, K., Hirata, T. \& Yamaguchi, A. (2002). The putative response regulator BaeR stimulates multidrug resistance of Escherichia coli via a novel multidrug exporter system, MdtABC. J Bacteriol 184, 4161-4167.

Pearson, J. P., Pesci, E. C. \& Iglewski, B. H. (1997). Roles of Pseudomonas aeruginosa las and rhl quorum-sensing systems in control of elastase and rhamnolipid biosynthesis genes. J Bacteriol 179, 5756-5757.

Posadas, D. M., Martin, F. A., Sabio y Garcia, J. V., Spera, J. M., Delpino, M. V., Baldi, P., Campos, E., Cravero, S. L. \& Zorreguieta, A. (2007). The TolC homologue of Brucella suis is involved in resistance 
to antimicrobial compounds and virulence. Infect Immun 75, 379-389.

Pumbwe, L. \& Piddock, J. V. (2002). Identification and molecular characterization of $\mathrm{CmeB}$, a Campylobacter jejuni multidrug efflux pump. FEMS Microbiol Lett 206, 185-189.

Ruzin, A., Keeney, D. \& Bradford, P. A. (2007). AdeABC multidrug efflux pump is associated with decreased susceptibility to tigecycline in Acinetobacter calcoaceticus-Acinetobacter baumannii complex. J Antimicrob Chemother 59, 1001-1004.

van Amsterdam, K., Bart, A. \& van der Ende, A. (2005). A Helicobacter pylori TolC efflux pump confers resistance to metronidazole. Antimicrob Agents Chemother 49, 1477-1482.

Edited by: P. Cornelis 